\title{
A DSO-level contract market for conditional demand response
}

\author{
Kok, Corey; Kazempour, Jalal; Pinson, Pierre
}

Published in:

Proceedings of IEEE PES PowerTech 2019

Link to article, DOI:

10.1109/PTC.2019.8810943

Publication date:

2020

Document Version

Peer reviewed version

Link back to DTU Orbit

Citation (APA):

Kok, C., Kazempour, J., \& Pinson, P. (2020). A DSO-level contract market for conditional demand response. In Proceedings of IEEE PES PowerTech 2019 IEEE. https://doi.org/10.1109/PTC.2019.8810943

\section{General rights}

Copyright and moral rights for the publications made accessible in the public portal are retained by the authors and/or other copyright owners and it is a condition of accessing publications that users recognise and abide by the legal requirements associated with these rights.

- Users may download and print one copy of any publication from the public portal for the purpose of private study or research.

- You may not further distribute the material or use it for any profit-making activity or commercial gain

- You may freely distribute the URL identifying the publication in the public portal

If you believe that this document breaches copyright please contact us providing details, and we will remove access to the work immediately and investigate your claim. 


\title{
A DSO-Level Contract Market for Conditional Demand Response
}

\author{
Corey Kok, Jalal Kazempour, Pierre Pinson \\ Technical University of Denmark, Centre for Electric Power and Energy, Kgs. Lyngby, Denmark \\ Email: \{corkok, seykaz, ppin\}@elektro.dtu.dk
}

\begin{abstract}
This paper proposes a fixed-term (e.g., monthly) Demand Response (DR) contract market. Based on the outcomes of this market, the Distribution System Operator (DSO) pays DR aggregators to modify power consumption within a fixed window each day. Two contract types are introduced: Scheduled contracts require the DR daily, while conditional contracts require the DR after an activation signal from the DSO. Asymmetric block offers, introducing integer variables, are used to model DR with a rebound effect, potentially causing the DR offers to clear at a loss for the aggregators. Without an activation cost for conditional contracts, the DSO has the incentive to dispatch DR, despite consumer discomfort exceeding grid security benefits. Thus, the proposed market incorporates side-payments. A numerical study shows that among all DR services considered, the proposed market determines the optimal service for the whole system, ensuring the profitability of each market participant.
\end{abstract}

Index Terms-Electricity market, fixed-term contract, conditional demand response, asymmetric block offer, side-payment.

\section{INTRODUCTION}

In the current European electricity markets, the market operators use a zonal model, ignoring the grid constraints within each bidding zone that often encompasses an entire country. The zonal market clearing brings challenges to both Transmission System Operators (TSOs) and Distribution System Operators (DSOs), who are responsible for the secure operation of their underlying grids. Ideas based on "flexibility markets" and "flexibility products" [1] as part of ensuring grid security, have recently been proposed, all relying on reservation and activation of flexible resources to meet the TSOs' and DSOs' needs (as well as the needs of other market participants, e.g., balance responsible parties). The majority of the flexible resources, especially Demand Response (DR) aggregators, is spread at the distribution level, and exploiting the flexibility of these resources may further worsen the DSOs' challenges depending on the utilisation of this flexibility. Several TSO-DSO coordination schemes have been recently proposed [2], [3], each model having their pros and cons. Among these schemes, the two main ones are based on either a common TSO-DSO flexibility market design, or two sequential flexibility markets, one for the DSO and another for the TSO, but this scheme requires a coordination on TSO-DSO interface flow.

In this paper, we take a different approach, proposing a fixterm (e.g., a monthly) contract market for the DSO. In this market, the DSO procures contracts for flexibility services from DR aggregators located at the distribution level, still leaving room for those aggregators to sell the flexibility left to other participants, e.g., TSO. The contracts procured may span specific time-periods during each day of the market horizon, e.g., the peak time periods only.

The benefits of using flexibility of the aggregation of thermostatically controlled loads are highlighted in [4] and [5]. One important observation in the functioning of these loads (and their aggregators in general) is that any load reduction causes a deviation from their steady-state operation, e.g., the set-point temperature of refrigerators. Thus, load reduction needs to follow a load increase to return to this steady state. This phenomenon is referred to as rebound (or kick-back) effect [6]. In the market context, this effect is modelled by defining two joint blocks (called response and rebound), one representing the load decrease and another corresponding to the load increase. The rebound block is following the response one with no time gap between the two blocks, and the combination of these two blocks is so-called as an asymmetric block offer [7]. By asymmetric, it means that the rebound and response blocks are not necessarily identical with respect to the time period and load quantity reduced/increased. The DSO benefit of utilising DR each day depends on the temperature and consumption patterns, which vary from day-to-day. For some days, the DSO benefit of dispatching DR may not justify the resulting consumer discomfort. Thus, we define two distinct DR services as the two products of the proposed DSOlevel contract market: Scheduled and conditional services. The former requires the DR units to provide their offer every day; and conditional services, where the DSO must provide an activation signal to dispatch the DR units. One may interpret the conditional demand response as "capacity reservation" for flexibility, which can be activated by DSO.

In this context, this paper designs a fixed-term contract market for DR, describing the types of aggregators expected to participate in this market, and how to fairly pay the aggregators that can provide demand response. Two main challenges arise when attempting to guarantee each DR aggregator's profitability. Firstly, allowing aggregators to offer asymmetric blocks introduces integer variables to the model. It is well documented that this can lead to revenue inadequacy, specifically the aggregators may incur a loss [8]. Secondly, for conditional services for DR, if there is no cost or limit on the number of times that the DSO can dispatch this DR, the DSO has an incentive to dispatch DR every day. 
Side-payments are introduced to tackle the challenges above, ensuring that each DR offer is revenue adequate. The market clearing guarantees that the optimal DR blocks (based on their offer-costs) are chosen, with the adjustment ensuring that each aggregator does not incur a loss from participating in this market. To prevent the over-dispatch of conditional services, aggregators can also set a dispatch cost where the payment to each aggregator is dependent on the number of activations. The proposed market design is being demonstrated in practice in the context of EcoGrid 2.0 project [9] in Bornholm island of Denmark.

The paper is laid out as follows. In Section II the interaction between aggregators and DSO is described. The DSO marketclearing tool is also formulated and described, with the ex-post adjustments required to ensure revenue adequacy. In Section III we present results from a case study that numerically validates the design of the proposed market and the ex-post adjustments. In Section IV we draw conclusions.

\section{MARKET DEFINITION}

We consider a DSO that buys flexibility services from DR aggregators within a competitive market. The services are in the form of fixed-term contracts, lasting roughly one month, that either schedule aggregators to provide DR every day, or require an activation signal from the DSO shortly before the DR service time period each day.

Once we have the DSO's bids and DR aggregators' offers, we need to determine the best combination of DSO bid and DR aggregator offers that maximises the profit to the system as a whole. We first present an optimisation model in Section II-B, then describe the side-payment process in Sections II-C and II-D.

\section{A. Description of Participants}

As each aggregator could also offer their flexibility in the TSO-level flexibility market [7], there is an opportunity cost to reserving flexibility in this DSO-level contract market and depends directly on the time and length of the DR service. If the weather is a significant factor determining whether the DSO dispatches the DR units, then the average day that a conditional service is activated can be significantly different to a scheduled service, possibly affecting the average dispatch cost to the aggregator. Thus, ahead of the market clearing and for each DR service $p \in \mathcal{P}$, the DSO must first declare the time periods $t \in \mathcal{T}_{p}$ when each DR unit must be available to provide their contracted service and the daily probability $\mathbb{P}_{p}$ of this activation.

One type of agents that we expect to participate in this market is aggregators $c \in \mathcal{C}$ of thermostatically controlled loads. These loads have a desired operating temperature and allow flexibility around this temperature for short periods of time. The aggregators can then use this flexibility to either provide real-time regulation services in TSO-level flexibility market or in this DSO-level market, which is primarily used to reduce power consumption peaks. Typically, these offers will come in the form of asymmetric block offers, where the

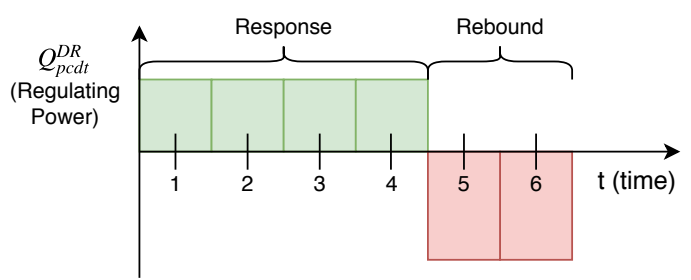

Fig. 1. A sample asymmetric block offer, including response (green) and rebound (red) blocks. In this specific example, the response block corresponds to an up regulation service (i.e. load decrease), while the rebound block corresponds to down regulation (load increase). Another type of block offer is the one whose response and rebound blocks provide down and up regulations, respectively.

load increase/decrease is followed by a load decrease/increase, as shown in Figure 1. We then map the entire block offer $d \in \mathcal{D}_{c}$ onto a parameter $\mathrm{Q}_{p c d t}^{\mathrm{DR}}$, giving the net response of the asymmetric block at each time $t$ (to meet DR service $p$ in the asymmetric block offered by aggregator $c$ ). In addition to loads with rebound, we also consider conventional load aggregators $i \in \mathcal{I}$, capable of providing demand response without rebound, that offer a maximum load reduction $\overline{\mathrm{P}}_{\text {pit }}$ at each time step $t$ for DR service $p$.

\section{B. Market Clearing as an Optimisation Problem}

The market-clearing algorithm, defined by the mixed-integer linear program (1) through (10e), determines the optimal combination of bids and offers for DR, minimising the overall system cost. The objective function is written as follows:

$$
\min _{\Xi} \sum_{p \in \mathcal{P}} R C_{p}+\sum_{p \in \mathcal{P}} \mathbb{P}_{p} D C_{p}
$$

where $\Xi$ includes the set of variables $p_{p i t}, r_{p c d}, m_{p c d}, s_{p t}, z_{p}$, and dummy variables $R C_{p}$ and $D C_{p}$. In the first term of (1), we have the reserve cost $R C_{p}$ corresponding to DR service $p$, defined in (2). This cost is independent of the number of days the service is dispatched and reflects any fixed costs associated for both the DSO and DR aggregator bidding into the DSO-level contract market. The main reserve cost will be the opportunity cost of the aggregator participating in the TSO-level flexibility market.

In the second term of (1), we have the expected dispatch cost $\mathbb{P}_{p} D C_{p}$, given by the daily probability of activation $\mathbb{P}_{p}$ (stated by the DSO) multiplied by net dispatch cost $D C_{p}$, which is defined in (3). Note that the daily probability of activation for scheduled services is equal to 1.0 , while for conditional services it is a value between zero and 1.0, and is stated before the market clearing by the DSO. The dispatch cost reflects the expected cost of redispatch in the TSO-level flexibility market and consumer discomfort. This objective is normalised to a single day. Thus, to calculate the actual cost, the optimal value obtained for (1) should be multiplied by the length of the contract, $\mathrm{L}$ in days. 
Equation (2) defines the cost of the reserve component of meeting the DR services.

$$
\begin{aligned}
R C_{p} & =\sum_{i \in \mathcal{I}} \sum_{t \in \mathcal{T}_{p}} \underbrace{\mathrm{C}_{p i t}^{\mathrm{R}, \mathrm{Con}} p_{p i t}}_{\text {conv. load cost }}+\sum_{c \in \mathcal{C}} \sum_{d \in \mathcal{D}_{c}} \underbrace{\mathrm{C}_{p c d}^{\mathrm{R}, \mathrm{DR}} r_{p c d}}_{\text {dem. resp. cost }} \\
& +\sum_{t \in \mathcal{T}_{p}} \underbrace{\mathrm{C}_{p t}^{\mathrm{R}, \mathrm{Reb}} s_{p t}}_{\text {rebound cost }}-\underbrace{\mathrm{C}_{p}^{\mathrm{R}, \mathrm{DSO}} z_{p}}_{\text {DSO benefit }} \quad \forall p \in \mathcal{P} .
\end{aligned}
$$

In the first term, we define the cost from conventional load aggregators, where the cost per $k W$ is given by $\mathrm{C}_{\text {pit }}^{\mathrm{R} \text {, Con }}$ (service $p$, conventional load aggregator $i$, time $t$ ) and the amount of conventional load reduction (in $k W$ ) is given by $p_{\text {pit }}$. In the second term, we have the cost from asymmetric block offers, where the cost per block $d$ (belonging to aggregator $c$ for service $p$ ) is given by $\mathrm{C}_{p c d}^{\mathrm{R}, \mathrm{DR}}$ and the number of blocks is given by integer variable $r_{p c d}$. In the third term, we have the cost to the DSO when aggregators utilise their allowed rebound, with the rebound cost per $k W$ is given by $\mathrm{C}_{p t}^{\mathrm{R}, \mathrm{Reb}}$ (service $p$, time $t$ ) and the amount of allowed rebound (in $k W$ ) is given by $s_{p t}$. In the final term, we subtract the benefit (utility) to the DSO for clearing the DR service, where the benefit of each DR service $p$ is given by $\mathrm{C}_{p}^{\mathrm{R}, \mathrm{DSO}}$ with binary variable $z_{p}$ indicating which DR service clears.

Similarly, (3) defines the cost from dispatch. Note that the cost parameters in (3) are different than those in (2), differentiated by a separate superscript ( $D$ instead of $R$ ).

$$
\begin{aligned}
D C_{p} & =\sum_{i \in \mathcal{I}} \sum_{t \in \mathcal{T}_{p}} \underbrace{\mathrm{C}_{p i t}^{\mathrm{D}, \mathrm{Con}} p_{\text {pit }}}_{\text {conv. load cost }}+\sum_{c \in \mathcal{C}} \sum_{d \in \mathcal{D}_{c}} \underbrace{\mathrm{C}_{p c d}^{\mathrm{D}, \mathrm{DR}} r_{p c d}}_{\text {dem. resp. cost }} \\
& +\sum_{t \in \mathcal{T}_{p}} \underbrace{\mathrm{C}_{p t}^{\mathrm{D}, \mathrm{Reb}} s_{p t}}_{\text {rebound cost }}-\underbrace{\mathrm{C}_{p}^{\mathrm{D}, \mathrm{DSO}} z_{p}}_{\text {DSO benefit }} \quad \forall p \in \mathcal{P} .
\end{aligned}
$$

Constraint (4) defines an upper limit $\overline{\mathrm{P}}_{\text {pit }}^{\text {Con }}$ on $p_{\text {pit }}$. This represents the amount of up regulation that each aggregator $i$ can provide at each time $t$ for each DR product $p$.

$$
p_{p i t} \leq \overline{\mathrm{P}}_{p i}^{\text {Con }} z_{p} \quad \forall p \in \mathcal{P}, i \in \mathcal{I}, t \in \mathcal{T}_{p} .
$$

According to the flexibility portfolio of aggregators within a given time period, each aggregator $c$ might be able to offer several asymmetric blocks, each with a different shape of response and rebound blocks. However, it can eventually deliver at most one of those block offers. Thus, we define binary variable $m_{p c d}$, indicating (for service $p$, aggregator $c$ ) which asymmetric block $d$ is chosen. For a given DR service indicated by $z_{p}$, constraint (5) allows each aggregator to offer multiple blocks into the market, knowing at most one of these blocks will be dispatched.

$$
\sum_{d \in \mathcal{D}_{c}} m_{p c d} \leq z_{p} \quad \forall p \in \mathcal{P}, c \in \mathcal{C} .
$$

Each asymmetric block offered into the DSO-level market can be split into multiple blocks. Constraint (6) defines the number of granular blocks $\mathrm{B}_{p c d}^{\mathrm{DR}}$ that make up the full utilisation of the asymmetric block $d$ (offered by DR aggregator $c$ for service $p$ ), where any integer up to $\mathrm{B}_{p c d}^{\mathrm{DR}}$ may clear the DSO-level market.

$$
r_{p c d} \leq \mathrm{B}_{p c d}^{\mathrm{DR}} m_{p c d} \quad \forall p \in \mathcal{P}, c \in \mathcal{C}, d \in \mathcal{D}_{c} .
$$

With the DSO able to submit multiple bids for demand response services $p$, each with their associated response requirements and benefit to the DSO, constraint (7) ensures that at most one of these services clears the market.

$$
\sum_{p \in \mathcal{P}} z_{p} \leq 1
$$

During rebound periods, constraint (8) limits the amount of rebound $s_{p t}$ to a maximum $\mathrm{D}_{p t}^{\mathrm{Reb}}$ of allowed rebound at each time $t$ for the cleared DR service $p$ (indicated with $z_{p}$ ).

$$
s_{p t} \leq \mathrm{D}_{p t}^{\mathrm{Reb}} z_{p} \quad \forall p \in \mathcal{P}, t \in \mathcal{T}_{p} .
$$

For each service $p$ and at each time $t$, constraint (9) enforces that the total load reduction provided by DR aggregators satisfies the minimum service requirement, accounting for the rebound effect. The parameter $\mathrm{D}_{p t}^{\mathrm{Reg}}$, set by the DSO, defines the minimum response required at each time $t$ for service $p$.

$$
\begin{gathered}
\sum_{i \in \mathcal{I}} p_{p i t}+\sum_{c \in \mathcal{C}} \sum_{d \in \mathcal{D}_{c}} \mathrm{Q}_{p c d t}^{\mathrm{DR}} r_{p c d} \geq \mathrm{D}_{p t}^{\mathrm{Reg}} z_{p}-s_{p t} \\
\forall p \in \mathcal{P}, t \in \mathcal{T}_{p} .
\end{gathered}
$$

Finally, constraints (10a)-(10e) constitute variable declarations.

$$
\begin{aligned}
p_{p i t} & \geq 0 \quad \forall p \in \mathcal{P}, i \in \mathcal{I}, t \in \mathcal{T}_{p} \\
r_{p c d} & \in \mathbb{Z}_{+} \quad \forall p \in \mathcal{P}, c \in \mathcal{C}, d \in \mathcal{D}_{c} \\
m_{p c d} & \in\{0,1\} \quad \forall p \in \mathcal{P}, c \in \mathcal{C}, d \in \mathcal{D}_{c} \\
z_{p} & \in\{0,1\} \quad \forall p \in \mathcal{P} \\
s_{p t} & \geq 0 \quad \forall p \in \mathcal{P}, t \in \mathcal{T}_{p} .
\end{aligned}
$$

The optimisation problem (1)-(10e) is a mixed-integer program, and therefore, we cannot derive dual variables for determining uniform market prices. One common solution for deriving prices from a mixed-integer problem, especially in the US markets, is to solve the original mixed-integer linear problem, and then to fix the values of integer and binary variables $\boldsymbol{r}, \boldsymbol{m}$ and $\boldsymbol{z}$ to those obtained from the original problem, which results in a linear and continuous problem. Then, the market prices can be obtained using the dual variables. However, it is now well-known that fixing integer and binary variables may yield unsupporting marketclearing prices, meaning that the market prices may not reflect all system costs, which may yield a negative profit for some market participants [8]. Therefore, a side (uplift) payment is required, as explained later in Section II-D.

\section{DSO Payment to Aggregator}

The original problem (1)-(10e) becomes a continuous problem by fixing integer and binary variables, and then the dual variable associated with (9) is treated as the market price. We denote this dual variable as $\pi_{p t}$, implying the market 
price of service $p$ at time $t$. Summing across the service time $\mathcal{T}_{p}$, equation (11a) gives the payment from the DSO to the conventional load aggregator $i$. Similarly, (11b) gives the payment to the aggregator $c$ :

$$
\begin{aligned}
T_{p i}^{\mathrm{Con}} & =\sum_{t \in \mathcal{T}_{p}} \pi_{p t} p_{p i t} \quad \forall p \in \mathcal{P}, i \in \mathcal{I} \\
T_{p c}^{\mathrm{DR}} & =\sum_{t \in \mathcal{T}_{p}} \pi_{p t} \sum_{d \in \mathcal{D}_{c}} \mathrm{Q}_{p c d t}^{\mathrm{DR}} r_{p c d} \quad \forall p \in \mathcal{P}, c \in \mathcal{C} .
\end{aligned}
$$

Assuming that all market participants are price-takers, i.e., all bids and offers represent the true utility and costs, the daily profit for each conventional load aggregator $i$, each DR aggregator $c$, and the DSO from each service $p$ is determined using (12a), (12b) and (12c), respectively:

$$
\begin{aligned}
W_{p i}^{\mathrm{Con}}= & T_{p i}^{\mathrm{Con}}-\sum_{t \in \mathcal{T}_{p}}\left(\mathrm{C}_{p i t}^{\mathrm{R}, \text { Con }}-\mathbb{P}_{p} \mathrm{C}_{p i t}^{\mathrm{D}, \mathrm{Con}}\right) p_{p i t} \\
& \forall p \in \mathcal{P}, i \in \mathcal{I} \\
W_{p c}^{\mathrm{DR}}= & T_{p c}^{\mathrm{DR}}-\sum_{d \in \mathcal{D}_{c}}\left(\mathrm{C}_{p c d}^{\mathrm{R}, \mathrm{DR}}-\mathbb{P}_{p} \mathrm{C}_{p c d}^{\mathrm{D}, \mathrm{DR}}\right) r_{p c d} \\
& \forall p \in \mathcal{P}, c \in \mathcal{C} \\
W_{p}^{\mathrm{DSO}}= & \sum_{t \in \mathcal{T}_{p}}\left(\mathrm{C}_{p}^{\mathrm{R}, \mathrm{DSO}}+\mathbb{P}_{p} \mathrm{C}_{p}^{\mathrm{D}, \mathrm{DSO}}\right) z_{p} \\
- & \sum_{t \in \mathcal{T}_{p}}\left(\mathrm{C}_{p t}^{\mathrm{R}, \mathrm{Reb}}+\mathbb{P}_{p} \mathrm{C}_{p t}^{\mathrm{D}, \mathrm{Reb}}\right) s_{p t} \\
- & \sum_{i \in \mathcal{I}} T_{p i}^{\mathrm{Con}}-\sum_{c \in \mathcal{C}} T_{p c}^{\mathrm{DR}} \quad \forall p \in \mathcal{P} .
\end{aligned}
$$

\section{Side-Payment}

There are two reasons that the DSO payments to conventional loads and DR aggregators based on (11a) and (11b) may need an adjustment. We explain below these two reasons and the process of determining the side-payment incurred by each of those reasons.

Firstly, as explained in Section II-B, fixing integer and binary variables may yield unsupporting prices $\pi_{p t}$, in the sense that DR aggregators may end up a negative profit, i.e., the values obtained for (12b) might be negative. In that case, the DSO should compensate their loss, and ensure that their profit will be eventually non-negative. It is outside the scope of this paper to address how the DSO can recover its sidepayments, but usually, it should distribute this cost in a fair manner among consumers.

Secondly, as explained in Section II-B, the DSO states the daily activation probability $\mathbb{P}_{p}$ for each service $p$ before the market clearing, whose value lies between zero and 1.0 (as before) for conditional services, and then the market participants offer to the market accordingly. However, the realised percentage of activation for conditional services, denoted by $\mathbb{Q}_{p}$, over the market horizon (e.g., a month) might be different than $\mathbb{P}_{p}$. Therefore, a side-payment is required; otherwise, the DSO tends to state a reduced value for $\mathbb{P}_{p}$, while the market participants are discouraged from offering in the market. We base our proposed side-payment on a pay-as-bid pricing scheme, meaning that for the difference of $\mathbb{Q}_{p}$ and $\mathbb{P}_{p}$, the market participants are paid/charged according to their price offers (which is equal to their true costs for the price-taker participants). The final payment from the DSO to conventional loads $i$ and DR aggregators $c$ is given below:

$$
\begin{aligned}
\widehat{T}_{p i}^{\text {Con }}= & \underbrace{T_{p i}^{\text {Con }}}_{\text {as in (11a) }}+\underbrace{\left(\mathbb{Q}_{p}-\mathbb{P}_{p}\right) \sum_{t \in \mathcal{T}_{p}} \mathrm{C}_{p i t}^{\mathrm{D}, \text { Con }} p_{p i t}}_{\text {side payment }} \\
& \forall p \in \mathcal{P}, i \in \mathcal{I} \quad \underbrace{T_{p c}^{\mathrm{DR}}}_{\text {as in (11b) }}+\underbrace{\left(\mathbb{Q}_{p}-\mathbb{P}_{p}\right) \sum_{d \in \mathcal{D}_{c}} \mathrm{C}_{p c d}^{\mathrm{D}, \mathrm{DR}} r_{p c d}}_{\text {side payment }} \\
& \forall p \in \mathcal{P}, c \in \mathcal{C} .
\end{aligned}
$$

Accordingly, the final daily profit of market participants and the DSO from each service $p$ is written below:

$$
\begin{aligned}
\widehat{W}_{p i}^{\mathrm{Con}}= & \widehat{T}_{p i}^{\mathrm{Con}}-\sum_{t \in \mathcal{T}_{p}}\left(\mathrm{C}_{p i t}^{\mathrm{R}, \mathrm{Con}}-\mathbb{Q}_{p} \mathrm{C}_{p i t}^{\mathrm{D}, \mathrm{Con}}\right) p_{p i t} \\
& \forall p \in \mathcal{P}, i \in \mathcal{I} \\
\widehat{W}_{p c}^{\mathrm{DR}}= & \widehat{T}_{p c}^{\mathrm{DR}}-\sum_{d \in \mathcal{D}_{c}}\left(\mathrm{C}_{p c d}^{\mathrm{R}, \mathrm{DR}}-\mathbb{Q}_{p} \mathrm{C}_{p c d}^{\mathrm{D}, \mathrm{DR}}\right) r_{p c d} \\
& \forall p \in \mathcal{P}, c \in \mathcal{C} \\
\widehat{W}_{p}^{\mathrm{DSO}}= & \sum_{t \in \mathcal{T}_{p}}\left(\mathrm{C}_{p}^{\mathrm{R}, \mathrm{DSO}}+\mathbb{Q}_{p} \mathrm{C}_{p}^{\mathrm{D}, \mathrm{DSO}}\right) z_{p} \\
- & \sum_{t \in \mathcal{T}_{p}}\left(\mathrm{C}_{p t}^{\mathrm{R}, \mathrm{Reb}}+\mathbb{Q}_{p} \mathrm{C}_{p t}^{\mathrm{D}, \mathrm{Reb}}\right) s_{p t} \\
- & \sum_{i \in \mathcal{I}} \widehat{T}_{p i}^{\mathrm{Con}}-\sum_{c \in \mathcal{C}} \widehat{T}_{p c}^{\mathrm{DR}} \quad \forall p \in \mathcal{P} .
\end{aligned}
$$

\section{NUMERICAL STUDY}

\section{A. Description of Case Study and Input Data}

We consider a case study in which the DSO desires to reduce peak power consumption, which occurs every day from 17:00 to 18:00. We also consider one hour before and one hour after the peak time period as the potential rebound hours, i.e., the DR aggregators may increase their consumption to some extent in these two rebound hours to be able to reduce their consumption in the peak time period. The DSO runs a monthly contract market, and four market participants offer to reduce their consumption during the peak hours. Two of these participants are the aggregators of conventional loads ( $i 1$ and $i 2$ ) with no rebound effects. The other two participants are aggregators $c 1$ and $c 2$, who model their rebound effect using asymmetric block offers.

We consider three distinct DR services in this market, but as enforced by (7), at most one of these services will be eventually traded. The first one is a scheduled DR service, referred to as Sched, implying that this service needs to be delivered everyday (i.e., $\mathbb{P}_{\text {Sched }}=1$ ). The other two services (denoted as Cond1 and Cond2) are both conditional, i.e., they are capacity reservations and are delivered only if the 


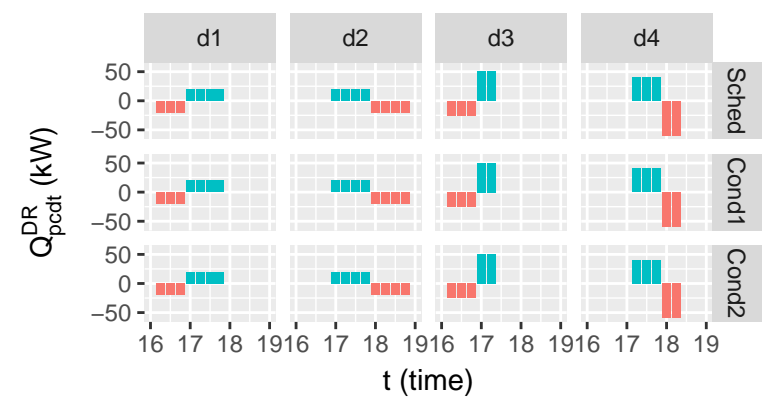

(a) Aggregator $c 1$

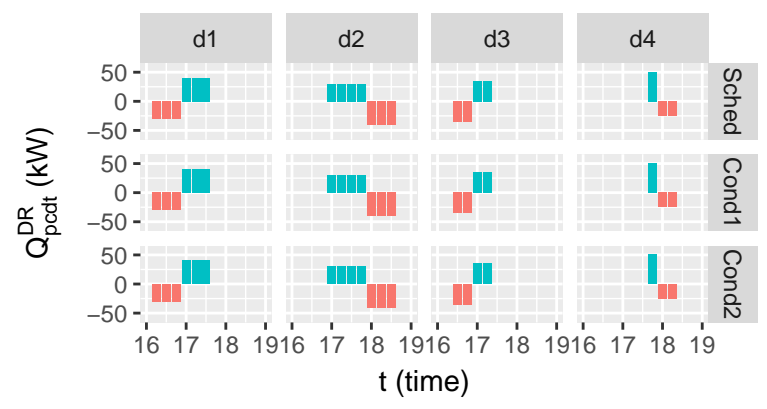

(b) Aggregator $c 2$

Fig. 2. Visualisation of the four asymmetric block offers (i.e., $d 1$ to $d 4$ ) of aggregator $c 1$ (upper plot) and aggregator $c 2$ (lower plot) for each DR service (Sched, Cond1, and Cond2).

TABLE I

RESERVE COST PER BLOCK, DISPATCH COST PER BLOCK, AND NUMBER OF DIVISIBLE BLOCKS

\begin{tabular}{ll|lll}
$c$ & $d$ & $\mathrm{C}_{p c d}^{\mathrm{R}, \mathrm{DR}}(€ / b k)$ & $\mathrm{C}_{p c d}^{\mathrm{D}, \mathrm{DR}}(€ / b k)$ & $\mathrm{B}_{p c d}^{\mathrm{DR}}(b k)$ \\
\hline$c 1$ & $d 1, d 2$ & 150 & 55 & 2 \\
$c 1$ & $d 3, d 4$ & 150 & 55 & 1 \\
$c 2$ & $d 1, d 2, d 3, d 4$ & 150 & 60 & 1
\end{tabular}

DSO activates them. The chance of these two services being activated is different, which is $\mathbb{P}_{\text {Cond } 1}=0.30$ and $\mathbb{P}_{\text {Cond } 2}=0.45$.

For each service, the aggregators $c 1$ and $c 2$ offer four asymmetric blocks ( $d 1$ to $d 2)$. The shape of these block offers is depicted in Figure 2, and their corresponding reserve and dispatch costs are given in Table I. In addition, the quantity and cost offers of conventional load aggregators $i 1$ and $i 2$ are provided in Table II. The benefit of the DSO from each service (i.e., DSO's bid prices) is given in Table III. The rebound cost of each service is also provided in Table IV. Finally, Table $\mathrm{V}$ provides the required service and the maximum allowed rebound for each DR service.

The optimisation problem (1)-(10e) is implemented in GAMS using CPLEX solver, and the code used is available in [10]. The CPU time for all cases is around 1 second.

\section{B. Results}

We now present the outcomes of the proposed market. Among the three services, Cond 2 is the one determined by the market clearing to be traded. The right-hand side plot of Figure 3, labelled MIP, shows the dispatch of the aggregators'
TABLE II

RESERVE COST $(€ / k W)$, DISPATCH COST $(€ / k W)$, AND MAXIMUM LOAD REDUCTION $(k W)$ FOR EACH CONVENTIONAL LOAD AGGREGATOR

\begin{tabular}{ll|lll}
$p$ & $i$ & $\mathrm{C}_{\text {pit }}^{\mathrm{R}, \text { Con }}$ & $\mathrm{C}_{\text {pit }}^{\mathrm{D}, \text { Con }}$ & $\overline{\mathrm{P}}_{\text {pit }}^{\text {Con }}$ \\
\hline Sched & $i 1$ & 2 & 4.0 & 50 \\
Sched & $i 2$ & 2 & 4.1 & 50 \\
Cond 1 & $i 1$ & 1 & 4.0 & 50 \\
Cond 1 & $i 2$ & 1 & 4.1 & 50 \\
Cond2 & $i 1$ & 1 & 4.0 & 50 \\
Cond2 & $i 2$ & 1 & 4.1 & 50
\end{tabular}

TABLE III

RESERVE AND DISPATCH BENEFIT FOR THE DSO FROM EACH DR SERVICE

\begin{tabular}{l|ll} 
Service $(p)$ & $\mathrm{C}_{p}^{\mathrm{R}, \operatorname{Res}}(€)$ & $\mathrm{C}_{p}^{\mathrm{D}, \mathrm{Res}}(€)$ \\
\hline Sched & 400 & 2400 \\
Cond 1, Cond 2 & 400 & 4000
\end{tabular}

TABLE IV

RESERVE AND DISPATCH REBOUND COST $(€ / k W)$ FOR EACH DR SERVICE

\begin{tabular}{ll|ll} 
Service $(p)$ & Time period $(t)$ & $\mathrm{C}_{p t}^{\mathrm{R}, \mathrm{Reb}}$ & $\mathrm{C}_{p t}^{\mathrm{D}, \mathrm{Reb}}$ \\
\hline Sched,Cond1,Cond2 & $16-16: 59,18-18: 59$ & 0 & 1
\end{tabular}

TABLE V

SERVICE REQUIREMENT AND REBOUND ALLOWED $(k W)$ FOR EACH DR SERVICE

\begin{tabular}{ll|ll} 
Service $(p)$ & Time period $(t)$ & $\mathrm{D}_{p t}^{\text {Reg }}$ & $\mathrm{D}_{p t}^{\text {Reb }}$ \\
\hline Sched, Cond1, Cond2 & $17-17: 59$ & 100 & 0 \\
Sched, Cond1, Cond2 & $16-16: 59,18-18: 59$ & 0 & 25
\end{tabular}

DR. Interestingly, the response block of aggregator $c 1$ provides up regulation, while such a service is provided by the rebound block of aggregator $c 2$. In other words, aggregator $c 1$ decreases consumption between hours 17 and 18, and then increases consumption in the next hour. In contrast, aggregator $c 2$ increases its consumption between hours 16 and 17, and then decreases consumption between hours 17 and 18. To highlight the importance of having binary and integer variables, the lefthand side plot of Figure 3, labelled by LP, shows the market outcomes if the integer constraints on $z$ and $\boldsymbol{r}$ are relaxed - as expected, the dispatch decisions are made up of linear combinations of the asymmetric blocks, no longer maintaining the asymmetric block shape.

Table VI presents the market prices for service Cond2. As explained in Section II-B, the market prices are in fact the dual variables $\pi_{p t}$ in problem (1)-(10e) when fixing the integer and binary variables. Table VII gives the daily profit of each market participant. As a benchmark, the second column of Table VII provides the daily profits obtained from the linear version of problem (1)-(10e) when the binary and integer variables are relaxed (not fixed) - this corresponds to the case illustrated in the left-hand side plot of Figure 3. As expected, no one incurs a negative profit. However, as given in the third column of Table VII, this may happen based on prices given in Table VI. In this case, we solve the original mixed-integer linear problem (1)-(10e), fix the values of binary and integer variables to their 


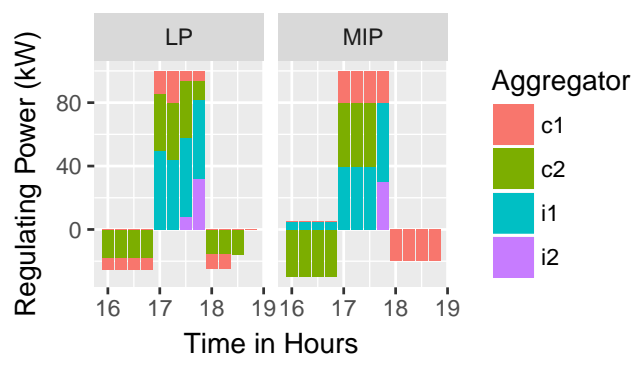

Fig. 3. Stacked upward and downward regulation services provided by DR aggregators. The negative values in time periods between hours 16 and 17 and between hours 18 and 19 represent load increase (downward regulation), while the positive values in the peak time period, i.e., between hours 17 and 18 show load decrease (upward regulation).

TABLE VI

MARKET PRICE FOR SERVICE Cond2, AFTER FIXING THE BINARY AND INTEGER VARIABLES AND THEN SOLVING THE LP MODEL

\begin{tabular}{l|r} 
Time period $(t)$ & $\pi_{p t}(€ / k W)$ \\
\hline $16: 00-16: 59$ & 2.80 \\
17:00-17:59 & 2.80 \\
18:00-18:59 & 0.45
\end{tabular}

TABLE VII

DAILY PROFIT (IN $€$ ) OF EACH PARTICIPANT AND THE SYSTEM AS A WHOLE IN EACH MODEL

\begin{tabular}{l|rrr} 
& \multicolumn{1}{|l}{ LP } & $\begin{array}{l}\text { MIP (before } \\
\text { side-payment) }\end{array}$ & $\begin{array}{l}\text { MIP (after } \\
\text { side-payment) }\end{array}$ \\
\hline$c 1$ & 0.00 & 14.15 & 14.15 \\
$c 2$ & 34.22 & -177.00 & 0.00 \\
$i 1$ & 5.20 & 2.25 & 2.25 \\
$i 2$ & 0.00 & 0.00 & 0.00 \\
DSO & 1176.74 & 1310.50 & 1133.50 \\
\hline Total & 1230.02 & 1149.90 & 1149.90
\end{tabular}

optimal values, and derive the dual variables $\pi_{p t}$ - the dispatch results of this case are the ones presented in the right-hand side plot of Figure 3. In this example, aggregator $c 2$ 's daily profit is $-€ 177$. Therefore, we require a side-payment, as presented in the last column of Table VII. In this case, after side-payment, the daily profit of aggregator $c 2$ increases to zero, while the DSO's benefit reduces.

We now consider the end of the market horizon (e.g., the end of the month), when the true proportion $\left(\mathbb{Q}_{C o n d 2}\right)$ of activation days for the conditional service is realised. We consider three cases, in which the realised proportion of days with DR activation is either 0.15 (i.e., lower than the DSO's expectation before market clearing), 0.45 (i.e., identical to the DSO's expectation), or 0.75 (i.e., higher than the DSO's expectation). As explained in Section II-D, we consider a side-payment based on a pay-as-bid auction. Accordingly, the aggregators providing service Cond 2 are charged in a case in which the realised proportion of days with DR activation is 0.15 , while they are paid in the case in which the realised proportion is 0.75 . Table VIII gives the final daily profit of all market participants before and after the side-payment. Before the side-payment, the aggregators' or the DSO's profit might be negative, becoming non-negative after the side-payment.
TABLE VIII

FINAL DAILY PROFIT (IN $€$ ) OF EACH PARTICIPANT AT THE END OF MARKET HORIZON, WHEN THE TRUE PERCENTAGE OF ACTIVATION FOR CONDITIONAL SERVICE $\left(\mathbb{Q}_{\text {Cond } 2}\right)$ IS REALISED

\begin{tabular}{l|l|rrrrr}
\multicolumn{2}{l|}{} & \multicolumn{5}{|c}{ Participant } \\
\hline Status & $\mathbb{Q}_{\text {Cond } 2}$ & $c 1$ & $c 2$ & $i 1$ & $i 2$ & DSO \\
\hline Before & 0.15 & 30.65 & 18.00 & 230.25 & 36.90 & -12.50 \\
Side & 0.45 & 14.15 & 0.00 & 2.25 & 0.00 & 1133.50 \\
Payment & 0.75 & -2.35 & -18.00 & -225.75 & -36.90 & 2279.50 \\
\hline After & 0.15 & 14.15 & 0.00 & 2.25 & 0.00 & 286.90 \\
Side & 0.45 & 14.15 & 0.00 & 2.25 & 0.00 & 1133.50 \\
Payment & 0.75 & 14.15 & 0.00 & 2.25 & 0.00 & 1980.10
\end{tabular}

\section{CONCLUSION}

This paper proposed a fixed-term (e.g., monthly) contract market, where the DSO can purchase load reduction services during peak hours from DR aggregators. Within the proposed services, we consider conditional ones, which are not necessarily delivered every day, and only activate upon the DSO's request. Although the proposed contract market provides the DSO with the optimal reservation of local flexibility resources, it might not be the optimal design for the whole electricity system, when considering the flexibility needs of the TSO. Therefore, the future work needs to study the impacts of the DSO's decisions on the performance of the TSO.

\section{ACKNOWLEDGMENT}

This work was supported by the Danish Energy Technology Development and Demonstration Programme (EUDP) through the project of EcoGrid 2.0 (64015-0082/EUDP). We thank Anubhav Ratha and Alexander Hermann (DTU) for suggestions.

\section{REFERENCES}

[1] J. Villar, R. Bessa, and M. Matos, "Flexibility products and markets: Literature review," Electric Power Systems Research, vol. 154, pp. 329340, 2018.

[2] A. Papavasiliou and I. Mezghani, "Coordination schemes for the integration of transmission and distribution system operations," in 20th Power Systems Computation Conference, Dublin, Ireland, 2018.

[3] J. Silva, J. Sumaili, R. J. Bessa, L. Seca, M. A. Matos, V. Miranda, M. Caujolle, B. Goncer, and M. Sebastian-Viana, "Estimating the active and reactive power flexibility area at the TSO-DSO interface," IEEE Transactions on Power Systems, vol. 33, no. 5, pp. 4741-4750, 2018.

[4] N. O'Connell, H. Madsen, P. Pinson, M. O'Malley, and T. Green, "Regulating power from supermarket refrigeration," in IEEE Innovative Smart Grid Technologies Conference, Europe, Istanbul, Turkey, 2014.

[5] K. Margellos and S. Oren, "Capacity controlled demand side management: A stochastic pricing analysis," IEEE Transactions on Power Systems, vol. 31, no. 1, pp. 706-717, 2016.

[6] L. A. Greening, D. L. Greene, and C. Difiglio, "Energy efficiency and consumption - the rebound effect - a survey," Energy policy, vol. 28, no. 6-7, pp. 389-401, 2000.

[7] N. O'Connell, P. Pinson, H. Madsen, and M. O'Malley, "Economic dispatch of demand response balancing through asymmetric block offers," IEEE Transactions on Power Systems, vol. 31, no. 4, pp. 2999-3007, 2016.

[8] R. P. O'Neill, P. M. Sotkiewicz, B. F. Hobbs, M. H. Rothkopf, and W. R. Stewart Jr, "Efficient market-clearing prices in markets with nonconvexities," European Journal of Operational Research, vol. 164, no. 1 , pp. 269-285, 2005.

[9] "EcoGrid 2.0." [Online]. Available: http://www.ecogrid.dk/en/home_uk

[10] "DSO Market Clearing Formulation." [Online]. Available: https: //github.com/coreykok/DSOMarketClearing 